\title{
How Do I Care for My Patients with...?
}

\author{
Thomas Kuehlein ${ }^{1}$, Ana Carvalho ${ }^{2}$, Catarina Viegas Dias ${ }^{3}$, David Rodrigues ${ }^{4}$ and Daniel Pinto ${ }^{4}$ \\ 1. Allgemeinmedizinisches Institut, Universitätsklinikum Erlangen, Universitätsstraße 29, Erlangen 91054, Germany \\ 2. Unidade de Saúde Familiar (USF) Loure Saudável, Loures 2670-487, Portugal \\ 3. Dafundo Family Medicine Unit, Dafundo 1495-713, Portugal \\ 4. Family Medicine Unit, NOVA Medical School/Faculdade de Ciências Médicas, Universidade Nova de Lisboa, Lisboa 169-056, \\ Portugal
}

Received: April 1, 2015 / Accepted: April 7, 2015 / Published: April 30, 2015.

\begin{abstract}
Introduction: Physicians want to know how they care for their patients. However, it can be challenging to describe the quality of their work. A basic question to ask would be: How do I care for my patients with a certain health condition? The authors wanted to find out whether they can answer this question for their patients suffering from osteoporosis with the support of their EHR (electronic health record). Materials and methods: Postmenopausal women having experienced a fracture were identified from the EHR. At the same time, criteria to assess the quality of care were defined following current guidelines. As it emerged that the EHR was unsuitable to fulfill the authors' purpose, the free software package "Epi Info 7" was used to create a template for data entry and analysis. Results: Of the 41 female patients identified with possible osteoporotic fractures, as many as $90 \%$ (37 patients) did not have any bone-density measurements recorded despite 39\% (16 patients) had been given a diagnosis of osteoporosis. Of the 16 patients with recorded osteoporosis, only two were prescribed antiosteoporotic medication. The EHR was largely inadequate to answer the question. Conclusion: "How do I care for my patients with...?" is a difficult question to answer when relying on conventional EHR. The resulting answer is worrying and opens up a lot of room for improvement on behalf of the EHR and the quality of care provided.
\end{abstract}

Key words: Quality of care, electronic health record, clinical governance, family practice.

\section{Introduction}

Many of the family physicians will have a sense of life comparable to that of a tennis player at the net with a ball machine gone mad in front of him (Fig. 1).

When we go home after a long working day, we know we have seen many different patients with many different problems and we are tired. Except from some striking cases, we will neither be able to tell what kind of problems we have been dealing with how often, nor will we be able to account for the quality of care we have given. The traditional way primary care physicians take account of the quality of their work is rooted in a subjective sense for the strength of their relationship with their patients. This sense is mainly based on the experience of their patients coming back

Corresponding author: Thomas Kuehlein, Ph.D., professor, research field: health services research. E-mail: Thomas.Kuehlein@uk-erlangen.de. presumably because they were content. We fully acknowledge the strength of the personal relationship between doctor and patient as an important marker of good quality of care. However, we suggest that this might not be sufficient and that we should try utilizing the data stored in our medical records to conduct audits in order to find out more about what we are doing all day long and whether we meet our own standards. Furthermore we believe that as physicians working in health care systems and spending money in a shared risk community, we are accountable not only to our patients, but also to society for what we do and how we spend this money.

Since the times of Hippocrates, doctors have documented their work. Today documentation is increasingly accomplished via EHRs (electronic health records). However, we probably do not tap the full potential of this new way of documentation. In 


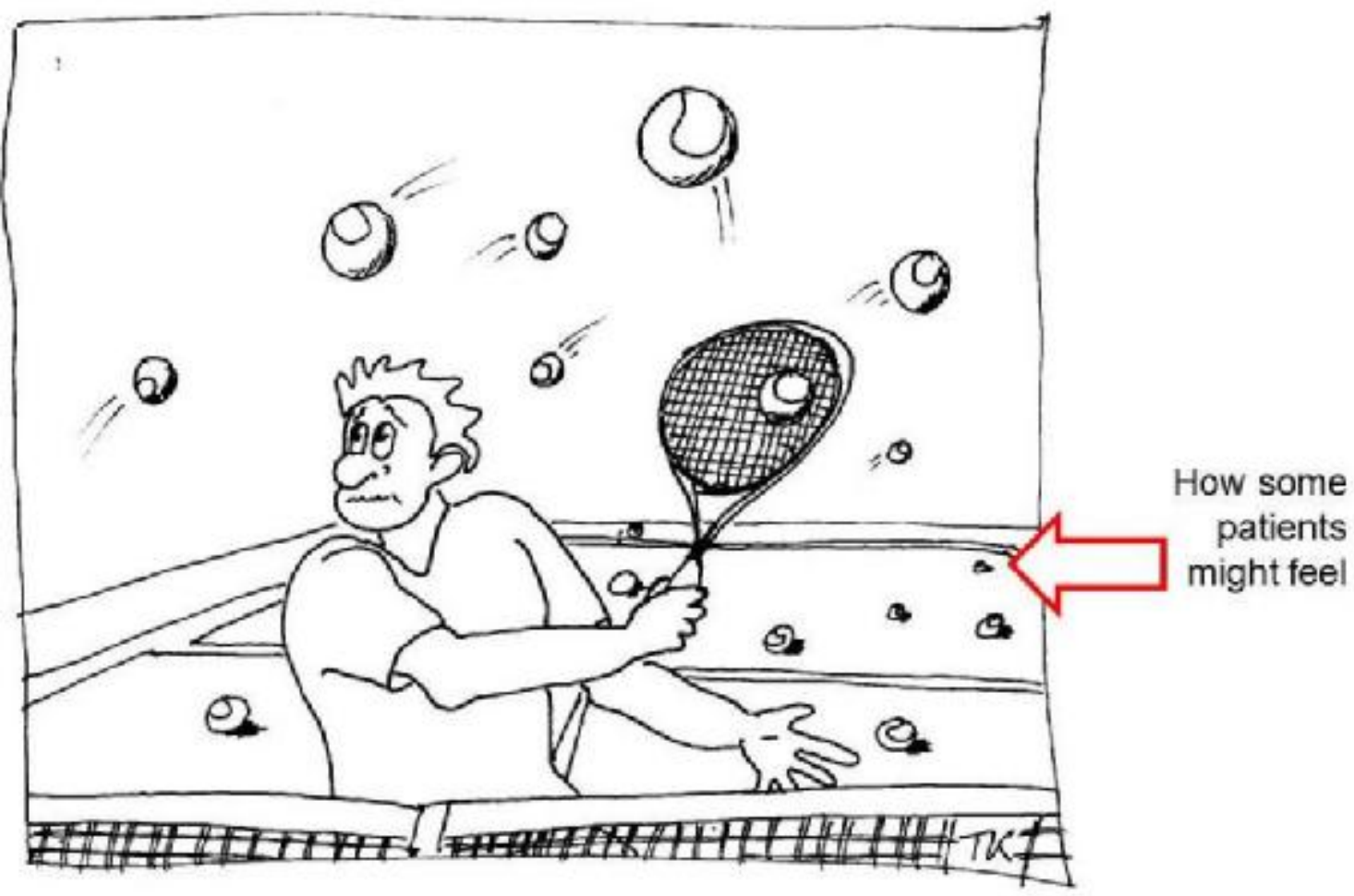

Fig. 1 Sense of life of a family physician.

Germany EHRs were primarily constructed for billing purposes plus the recording of narrative information similar to the former paper based health record. This may be sufficient-though far from optimal-to store information for single patients.

The basic question of the physician when asking for the quality of care she provides would be: "How do I care for my patients with...?"-with a certain health problem to be inserted in order to inquire the quality of care for specific patient groups. To describe the quality of care for certain groups of patients statistically, information must be structured and classified in order to be retrievable. The process of classification inevitably leads to a loss in detail concerning the individual patient. On the other hand, it is the only way to make things countable and to avoid mixing up apples with oranges. Even if God has not created all apples or oranges equal, they are at least similar in some respect. We assume that most EHRs do not allow putting down information in a sufficiently structured and classified way and are therefore unsuitable to answer the question stated above satisfactorily.

As a first exercise we wanted to know how far we could get in exploring the quality of care for our patients with osteoporosis in our EHRs. The focus of this exercise was not so much the actual care for osteoporotic patients, but the technical procedures in our EHRs. The results were presented and discussed in a workshop at the WICC-Open Day, a local conference one day before the yearly meeting of the WICC (Wonca International Classification Committee) in Lisbon, Portugal. Wonca is the world organization of family physicians. WICC works mainly on the maintenance and development of the ICPC (International Classification of Primary Care). The present article is a report of this workshop.

Four of us attempted finding an answer to our question in three different EHRs, one in Germany, the other two in Portugal. Due to space limitation we will only sketch the experiences with of one of them-the German EHR. 


\section{Materials and Methods}

\subsection{Creating a Patient List}

The first task would be to create a patient list. Osteoporosis is primarily defined as a bone density of less than-2.5 standard deviation of the mean of women between the ages of 20 and 29 [1]. However, the positive predictive values for fractures based on this arbitrary cut-off alone are fairly low. Having experienced a fracture without adequate trauma, the diagnosis changes from osteoporosis to severe or manifest osteoporosis. There is evidence that antiosteoporotic drugs can prevent osteoporotic fractures and that they might be considered for some patients [2]. The NNT (numbers of patients needed to treat) to avoid one future fracture are fairly high (NNT around 100/year [3]) for patients with low bone density only, but fall markedly with rising numbers of other independent risk factors for fractures and the most after the first osteoporotic fracture. This suggests that a quality improving process should focus in the first place on an area with the highest potential for meaningful benefit-patients with manifest osteoporosis. We focused on women, as osteoporotic fractures are far more frequent in women than in men. Only looking for women with the diagnosis "severe osteoporosis" would miss a great number of cases. Many patients with fractures probably never had their bone density measured.

Therefore the case finding strategy chosen followed a pragmatic approach. We searched in the EHR for all women older than 50 years with the letter-string "...fracture..." in the diagnoses. In a rural group practice caring for about 3,000 patients seen per quarter of the year, this case finding strategy resulted in a list of 70 patients. These 70 patients were hand searched. The German modification of the ICD-10-GM (International classification of diseases) allows the coding of diagnoses as "secured", "suspected" or "ruled out". Cases with the latter two were excluded. When taking into account patients with presumably non-osteoporotic fractures such as finger fractures, 29 patients could be excluded. The final search resulted in a list of 41 most likely postmenopausal women with fractures possibly due to osteoporosis.

\subsection{Data Capture and Criteria to Assess Quality of} Care

For data capture and processing we used the software package "Epi Info 7"-which is provided for free by the US "Centers for Disease Control and Prevention" [4]. The software tool allows for creating templates to capture information resulting in a database (Fig. 2). Furthermore "Epi Info 7" allows for analyzing the data. The first part of the template gives pseudonymized information about the patient such as age, BMI (body mass index), the number of medications taken as a proxy for multimorbidity and the date of data retrieval. Then we wanted to know whether the diagnosis of osteoporosis or manifest osteoporosis was documented in the record of the patient. We also looked for the diagnoses "osteoporosis suspected" and "osteoporosis ruled out". The next step was to assess whether any kind of bone density measurement had been performed and what the resulting T- and Z-scores were. Finally we were looking for the kind of antiosteoporotic therapy the patients had been prescribed.

As in our own HER-and probably in many others-information is not sufficiently stored in a standardized, coded and automatically retrievable way, it turned out, that the EHR was not of much help to answer our question. The template had to be filled in by hand. Diagnostic processes like bone density measurement are performed outside the primary care practices and the results are entered in the EHR by storing the scanned specialist letters. Prescribed medication is captured by the EHR but cannot be aggregated to higher levels of the ATC (anatomical-therapeutic-chemical)-classification of WHO as for example "bisphosphonates". What can be retrieved is date of birth (to calculate age by years) and 


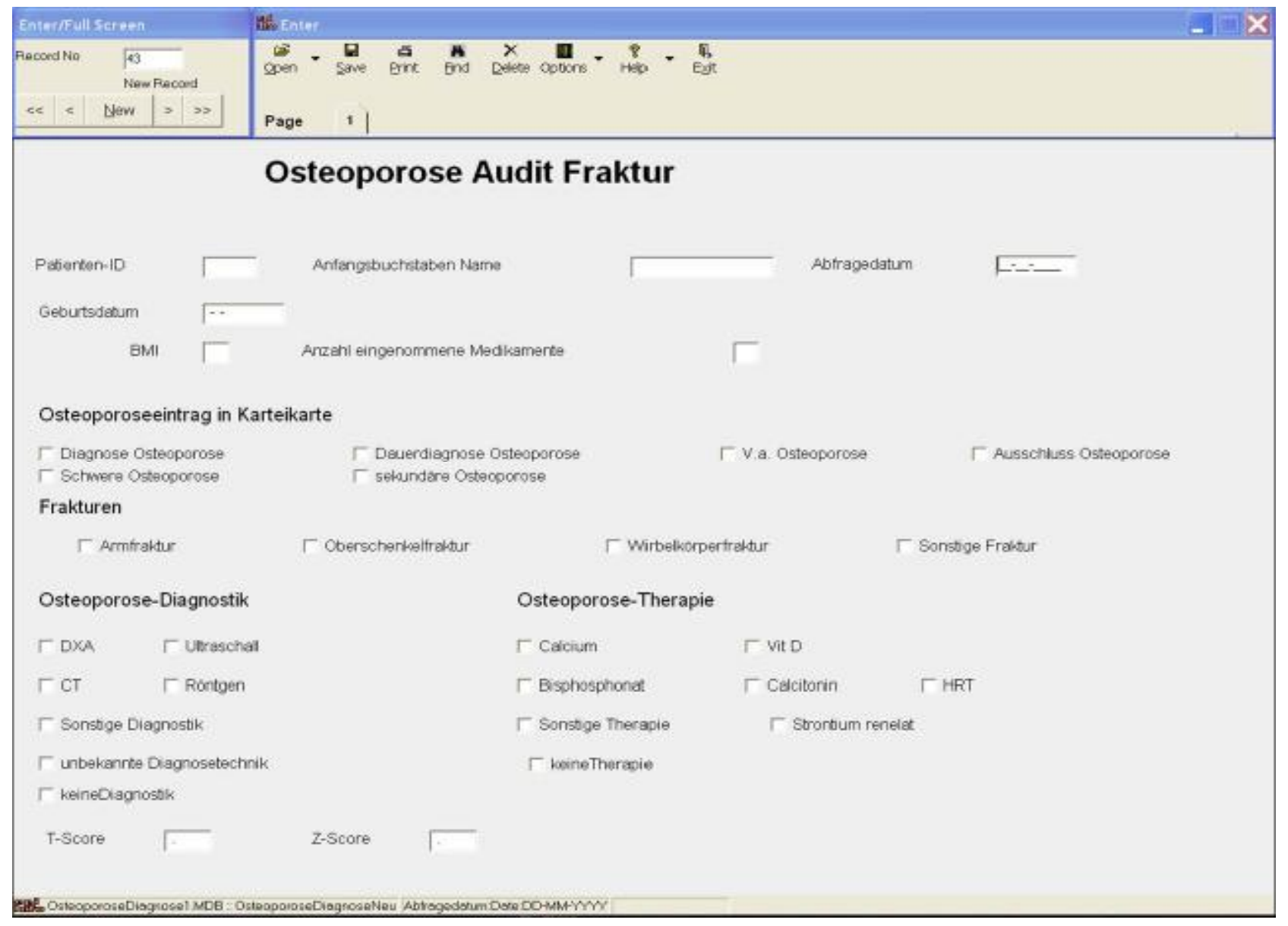

Fig. 2 Epi Info template to capture data about the quality of care for osteoporotic patients.

diagnoses coded with ICD-10-GM. BMI if documented at all can be identified by looking for certain line identifiers in the EHR but mostly weight and height are documented narratively not allowing electronic retrieval.

\section{Results}

\subsection{Patient's Characteristics}

There were 41 female patients with possible osteoporotic fractures with a mean age of 77.8 years (SD 10.5). The mean number of medications taken was 7.3 (min. 0; max. 18). BMI was recorded for only 14 patients. Their mean BMI was 28.4 (SD 5.6).

\subsection{Fractures}

A total of 49 fractures was found in the 41 patients. The fracture sites were as follows: 13 femoral, 13 arm,
11 vertebral, and 12 other location.

\subsection{Diagnoses}

Of the 41 patients 16 (39\%) had been diagnosed with osteoporosis. Furthermore, osteoporosis had been suspected in one patient and excluded in another. Of the 16 patients diagnosed with osteoporosis, only 11 had this diagnosis recorded in the problem list. As all patients had experienced a fracture, the correct diagnosis should not have been "osteoporosis" but "severe" or "manifest osteoporosis" instead. We found this entry in only one of the patients. The diagnosis "secondary osteoporosis" was recorded for one patient.

\subsection{Diagnostic Procedures}

No specific diagnostic procedures for osteoporosis could be found in 39 of the 41 patients (95\%). This means also that for 14 of the 16 patients who had been 
diagnosed with osteoporosis no concrete diagnostic procedure could be identified. As a consequence, it remained unclear where the diagnosis came from. In all, only one of the 41 patients had a DXA measurement recorded; one was diagnosed with a CT-bone-density technique and none with ultrasound. For two patients there were $\mathrm{T}$ - or Z-scores given in letters of orthopedic surgeons but without stating the place or technique of measurement. In two patients a radiologist had diagnosed osteoporosis after a plain $\mathrm{x}$-ray, which is not appropriate.

\subsection{Therapy}

Only seven of the patients had been prescribed calcium and vitamin D. For patients with manifest osteoporosis the German health care insurances pay for calcium and vitamin D. However, it is possible that some patients buy these drugs over the counter as they are not expensive. If the diagnoses are correct and the fractures resulted from inadequate trauma, only two of the 16 patients with manifest osteoporosis would have received adequate treatment with bisphosphonates; one received strontium ranelate. Hormone replacement therapy or calcitonin were not among the medications prescribed.

\subsection{Summary of the Results}

Of the 41 patients with possible osteoporotic fractures only one had recorded the appropriate DXA bone-density measurement. Accepting diagnostic measures such as the one case of CT-measurement and the two cases of Z- and T-scores given without stating the technique and place, no appropriate diagnostics were recorded in 37 of 41 patients (90\%). Of the 16 patients with manifest osteoporosis only two received a bisphosphonate and one strontium ranelate.

The attempts to answer the question within the two Portuguese EHRs yielded similar kinds of problems. Also, the results did not differ much.

\section{Discussion}

The results of our little exercise can only be seen as a full catastrophe. The problems are twofold:

(1) EHRs do not easily allow answering the simple but important question "How do I care for my patients with...?"

(2) Once the answer is reached in a quite cumbersome way, the results in case of patients with possible osteoporotic fractures seem quite disillusioning.

However:

(3) In case of osteoporosis the answer to the question "How should I care for my patients with ...?" is not easy as well.

Concerning the first problem: The reason why many EHRs are unsuitable to answer our question is probably that family physicians rarely ask this kind of question. They have only been trained to care for and focus on one individual patient after the other. However, in the natural "messiness of general practice" [5] supposedly many relevant aspects of good quality of care get lost. Within health care systems there have been attempts to answer the question of the quality of care for certain frequent health problems and also attempts to induce quality improvement. Most of them came top-down on us physicians and had limited effects. In Germany, it was the so called "DMPs (disease management programs)" for coronary heart disease, diabetes, asthma and COPD. The DMP documentation process is quite cumbersome and is therefore often delegated to practice assistants. The data are subsequently sent to external data processing centers perceived as administrative supervisory bodies. Unfortunately, the results rarely find their way back into the daily workflow of physicians resulting in only poor improvement of uncertain clinical benefit $[6,7]$. We suggest that the central problem with these programs is their lack of ownership for the caring teams. Professionalism in general is centrally founded on self-reflection and self-control on the one side and accountability on the other [8]. If we as practicing physicians are not able to statistically describe the quality of care for our patients, there is no reason why 
society should trust that quality is good. The results of our little exercise suggest that there is much room for improvement. A good EHR might not only allow for analyzing the quality of care we deliver but also support us in becoming better. For example, it might remind us to perform bone density measurement if we enter the diagnosis of a fracture for women over 50 or older men. If the central means to answer our question and also to support decision making is the EHR, we should demand from the software companies to deliver appropriate tools for our work and should be willing to pay for them.

Concerning the second problem: Even being quite cumbersome, it was possible to answer our question. The results seem disastrous at first sight. If we call the process described above "clinical governance within the practice", it must be said that all that clinical governance can achieve is to reduce the frequency of simply forgetting to decide. Decisions can only be taken individually, not for patient groups. Preferably they are taken as shared decisions together with the patient. The benchmarks are certainly never $100 \%$. However, it seems quite obvious that the results of our audit are far below any acceptable level of quality. If in almost $90 \%$ of women with possibly osteoporotic fractures no bone-density measurement is recorded, this is a clear sign of a lack of quality of care for these patients independent of the results of these measurements and the therapeutic consequences following. The only little solace might be that the failure probably is rooted more in the system than in the single physician. These patients had been seen by a hospital physician as the fracture happened and as Germany does not have a separation between primary and secondary care, most of the patients were probably seen by othopedic surgeaons and gynecologists in ambuatory care as well. All of them failed to look for osteoporosis after the fractures or at least to inform each other. Why might this be the case? Why does nobody know about the standard of care for possibly osteoporotic fractures? Again: The question is asked for a group of patients. The traditional focus of physicians is the single patient, if not even the single organ of the single patient. We do not suggest that care should only focus on groups with specific problems instead of individuals. However, we hypothesize that the shameful answer to our question of how we care for a specific patient group will result in better care for the individual patient. There is one more explanation for our results: documentation might be worse than actual care. However, we suppose that even if this is possible-although we think it is improbable-there is a high correlation between the quality of care and the quality of documentation.

Concerning the third problem: The answer to the question "How should I care for my patients with ...?" is neither easy nor straightforward. What might be clearly indicated for one patient might be of little or no value for another patient. In a Cochrane review by Wells et al., the results of randomized controlled trials on the bisphosphonate alendronate for patients with low bone density not having experienced a fracture yet, showed only negligible effects [9]. Also Wells et al. give a "best estimate of what happens to women that ... have already had a fracture" as follows:

"Fracture of the spine

- 12 out of 100 women had a fracture when taking a placebo

- 6 out of 100 women had a fracture when taking alendronate

Fracture in the hip or wrist

- 2 out of 100 women had a fracture when taking a placebo

- 1 out of 100 women had a fracture when taking alendronate

Fractures in bones other than the spine

- 9 out of 100 women had a fracture when taking a placebo

- 7 out of 100 women had a fracture when taking alendronate"

These small benefits need to be counterbalanced by possible side effects. Furthermore, the beneficial 
results seem to be strongly threatened by bias [10]. The largest effect is for women who have experienced vertebral fractures. While an antiosteoporotic medication might be meaningful for a relatively young patient with low comorbidity, the same medication might be superfluous for a very old person with many different and more threatening other conditions. Also multimorbidity, socio-economic status and other factors may influence a physician's decision whether or not to prescribe a treatment. Therefore, we also need EHRs able to capture other bits of information that may be outside the scope of a specific disease, but impact the physician's decision process.

For some of the bone measurements that will have to be performed after the audit, the results will be normal. For some of the patients with osteoporosis who had experienced a fracture, the small effects of bisphosphonates especially for non-vertebral fractures might result in an individual decision not to prescribe them. In all, this might result in a net effect of changing care for only very few patients. However, even if the result might be the same, there is a substantial difference between having decided in a process of shared decision making not to treat and simply having forgotten to decide.

\section{Conclusion}

We propose to ask the question "How do I care for my patients with...?" regularly and to try to answer it with the help of our EHRs. We must face the results even if they might be painful for us, hopefully with the effect of lesser pain for our patients in the future. It is due to our lack of questioning why our EHRs are so insufficiently equipped to answer it. We hypothesize that clinical governance will have better effects if conducted in ownership as "reflective practitioners" [11]. Whether this is a utopian dream depends on us.

\section{References}

[1] Kanis, J. A. On behalf of the World Health Organization
Scientific Group. 2007. "Assessment of Osteoporosis at the Primary Health-Care Level." Edited by WHO Geneva, Technical Report Series 843.

[2] NICE technology appraisal guidance 160. 2008. "Alendronate, Etidronate, Risedronate, Raloxifene and Strontium Ranelate for the Primary Prevention of Osteoporotic Fragility Fractures in Postmenopausal Women (Amended)." Accessed March 21, 2015. http://www.nice.org.uk/guidance/ta160/resources/guidanc e-alendronate-etidronate-risedronate-raloxifene-and-stron tium-ranelate-for-the-primary-prevention-of-osteoporotic -fragility-fractures-in-postmenopausal-women-amendedpdf

[3] Liberman, U. A., Weiss, S. R., Bröll, J., Minne, H. W., Quan, H., Bell, N. H., Rodriguez-Portalez, J., Downs, R. W., Dequeker, J., Favus, M., Seeman, E., Recker, R. R., Capizzi, T., Santora, A. C., Lombardi, A., Shah, R. V., Hirsch, L. J., and Karpf, D. B. for the Alendronate Phase III Osteoporosis Treatment Study Group. 1995. "Effect of Oral Alendronate on Bone Mineral Density and the Incidence of Fractures in Postmenopausal Osteoporosis." N. Engl. J. Med. 333: 1437-43.

[4] Center of Disease Control. "Epi Info 7." Free software download at: http://wwwn.cdc.gov/epiinfo/7/.

[5] Berg, M., and Mol, A. 1998. "Differences in Medicine: An Introduction." In Differences in Medicine-Unraveling Practices, Techniques and Bodies, edited by Berg, M., and Mol, A. Duke University Press, Durham.

[6] Miksch, A., Laux, G., Ose, D., Joos, S., Campbell, S., Riens, B., and Szecsenyi, J. 2010. "Is There a Survival Benefit within a German Primary Care-Based Disease Management Program?” Am. J. Manag Care 16: 49-54.

[7] Linder, R., Ahrens, S., Köppel, D., Heilmann, T., and Verheyen, F. 2011. "The Benefit and Efficiency of the Disease Management Program for Type 2 Diabetes." Dtsch Arztebl Int. 108: 155-62.

[8] Abbott, A. 1988. The System of Professions: An Essay on the Division of Expert Labor. Chicago: University of Chicago Press.

[9] Wells, G. A., Cranney, A., Peterson, J., Boucher, M., Shea, B., Welch, V., Coyle, D., and Tugwell, P. 2008. "Alendronate for the Primary and Secondary Prevention of Osteoporotic Fractures in Postmenopausal Women." Cochrane Database of Systematic Reviews 13 (1) No.: CD001155. DOI: 10.1002/14651858.CD001155.pub2.

[10] Therapeutics Initiative. 2011. "A Systematic Review of the Efficacy of Bisphosphonates." Therapeutics Letter 83: $1-2$.

[11] Schön, D. A. 1983. The Reflective Practitioner-How Professionals Think in Action. New York: Basic Books. 